\title{
Speed Control of a Permanent Magnet Synchronous Motor Using Predictive Current Control
}

\author{
Esteban J. Fuentes, Jose Rodriguez, \\ César Silva, Sergio Díaz \\ Departamento de Electrónica \\ Universidad Técnica Federico Santa María \\ Valparaíso, Chile \\ Email: cesar.silva@usm.cl
}

\author{
Daniel E. Quevedo, \\ School of Electrical Engineering and Computer Science \\ The University of Newcastle \\ Callaghan, Australia \\ Email: dquevedo@ieee.org
}

\begin{abstract}
This paper presents the use of a predictive technique to control the currents in a PMSM, driven by a voltage source inverter. This method allows for a high quality current control without using linear controllers and modulators, resulting in a conceptually simple solution.

Simulation an experimental results confirm the good performance of the drive controlled by this method.
\end{abstract}

\section{INTRODUCTION}

Permanent magnet synchronous motors (PMSM) have high torque and power density, and excellent dynamic characteristics. Thus, they are specially well suited for servo drives and applications where size and weight are major constraints (e.g., automotive and aerospace industry). The typical construction of a PMSM consist of a three phase stator winding and a solid iron rotor with magnets attached to its surface or inserted into the rotor body. This construction results in a magnetic field fixed to the rotor position. Since such machines are not capable of directly starting from the mains, excitation by voltage source inverters (VSI) controlled by field orientation is required. Control techniques such as vector control [1] or direct torque control (DTC) [2] are standard for this type of drives.

The discrete nature of the output voltage in a VSI inverter has motivated some authors to use finite alphabet predictive control techniques for current control in this type of power converters [3]. These techniques are also applicable to machine drives, and allow one to replace the inner current control loop in vector control, or the inner torque and flux control loops in DTC techniques [4]. Similar approaches have been proposed in [5] [6]. Predictive control is a conceptually simple technique based on the prediction of the effects of all possible actuation values on the variables to be controlled, and the subsequent choice of the best actuation value, as measured by an appropriate cost function [7]. This method allows one to control the motor currents without the requirement of linear controllers and modulators.

The control scheme proposed in this paper considers a predictive controller for the inner current control of a PMSM, driven by a two level VSI. The current control is made in a $d q$ frame, such as in standard vector control. The control goals of this controller are current reference tracking, current amplitude limitation and torque by ampere ratio optimization. The current reference for this controller is given by an external PI speed controller.

The performance of the proposed method is tested through simulation and experimental results, which are obtained in a $4[k W]$ laboratory set up.

In this article, an appropriate model of the drive, including machine and power converter, is developed in section II. In section III the predictive control strategy is presented. Simulation and experimental results are presented in sections IV V, respectively. Finally, conclusions highlighting the advantages of the proposed method are given in section VI.

\section{THE DRIVE MODEL}

In order to implement a predictive control strategy for a given system, a discrete model, taking into account all the relevant dynamics, must be developed. Furthermore, for one step finite alphabet model predictive control (FAMPC), this model must include the set of discrete actuations available and must have direct feedthrough from the input of the system to the relevant states to be controlled.

In this section, the continuous time state space model for the PMSM is developed. For the purpose of current control, this model only need to include the electrical dynamics of the machine stator. This model is then discretized in order to obtain a model suitable for FAMPC formulation (see, e.g.,[8]). Finally, the set of discrete actuations is obtained from the vector 
representation of the conventional three phase voltage source inverter.

\section{A. Machine Model}

Using well-known space vector notation [9], the dynamics of the stator of the PMSM can be written as

$$
\vec{v}_{s}=R_{s} \vec{i}_{s}+\frac{d \vec{\psi}_{s}}{d t}
$$

where $R_{s}$ is the winding resistance, and $\vec{v}_{s}, \vec{i}_{s}$ and $\vec{\psi}_{s}$ are the stator voltage vector at the machine terminals, the stator current vector and the flux linked by the stator windings, respectively.

The stator flux linked by the stator windings $\vec{\psi}_{s}$ has two sources: the field produced by the rotor magnets, which is dependent on the rotor position $\theta_{r}$, and the self-linked flux due to the current flowing through the stator windings. So, if $L_{s}$ denotes the stator selfinductance, we have that

$$
\vec{\psi}_{s}=L_{s} \vec{i}_{s}+\psi_{m} e^{j \theta_{r}},
$$

where $\psi_{m}$ is the magnitude of the flux due to de rotor magnets. Replacing (2) into (1), the stator dynamics can be written as

$$
\vec{v}_{s}=R_{s} \vec{i}_{s}+L_{s} \frac{d \overrightarrow{i_{s}}}{d t}+j \psi_{m} \omega_{r} e^{j \theta_{r}}
$$

where $\omega_{r} \triangleq \frac{d \theta_{r}}{d t}$ is the rotor electrical angular frequency.

Conventionally, vector control of synchronous machines is implemented in a rotatory frame $(d q)$ which is oriented with the rotor magnetic field axis [1]. Using this frame of reference has the advantage of transforming sinusoidal variables into DC values. This yields a model that is similar to that of a DC machine and, hence, to a conceptually simple drive implementation. In this frame, each current component of the synchronous machine has a clear physical meaning: the imaginary component $i_{s q}$ is proportional to the torque, while the real component $i_{s d}$ is proportional to reactive power. Although the implementation of a predictive control strategy could use any reference frame, the rotatory $d q$ frame is preferred in this work for its simple physical interpretation. Transforming the stator dynamics equation into the $d q$ coordinate system results in

$$
\vec{v}_{s}^{(r)}=R_{s} \vec{i}_{s}^{(r)}+L_{s} \frac{d \vec{i}_{s}^{(r)}}{d t}+j \omega_{r} \vec{i}_{s}^{(r)}+j \psi_{m} \omega_{r},
$$

where superscript ${ }^{(r)}$ denotes variables in the $d q$ frame.

The relation between the stator electrical variables can be better understood in figure 1, where a vector diagram of the stator variables is presented.

This model can be synthesized in the following state

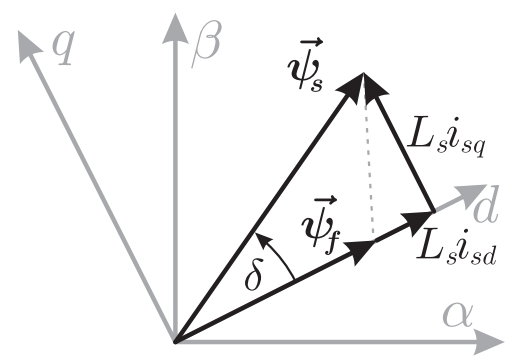

Fig. 1. Vector diagram of the stator electrical variables of the PMSM.

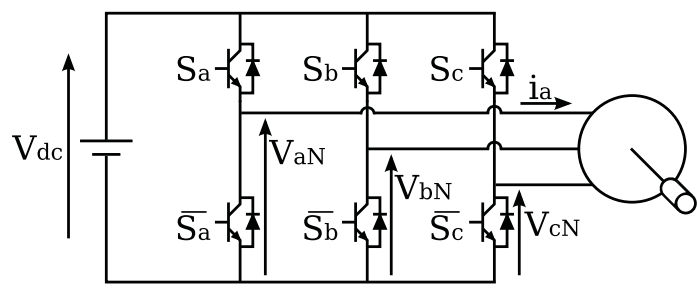

Fig. 2. Two level voltage source inverter

space model:

$$
\frac{d x(t)}{d t}=A(t) x(t)+B u(t)+d(t),
$$

where

$$
\begin{aligned}
x & \triangleq\left[\begin{array}{ll}
i_{s d} & i_{s q}
\end{array}\right]^{T} \\
u & \triangleq\left[\begin{array}{ll}
v_{d} & v_{q}
\end{array}\right]^{T} \\
d(t) & \triangleq\left[\begin{array}{ll}
0 & -\frac{\psi_{m}}{L_{s}} \omega_{r}(t)
\end{array}\right]^{T} \\
A(t) & \triangleq\left[\begin{array}{cc}
-\frac{1}{\tau_{s}} & \omega_{r}(t) \\
-\omega_{r}(t) & -\frac{1}{\tau_{s}}
\end{array}\right] \\
B & \triangleq\left[\begin{array}{cc}
\frac{1}{L_{s}} & 0 \\
0 & \frac{1}{L_{s}}
\end{array}\right]
\end{aligned}
$$

where $\tau_{s} \triangleq \frac{L_{s}}{R_{s}}$ is the stator time constant.

In order to implement a predictive model for the FAMPC algorithm, a model which is discrete in nature is needed. We choose to adopt an Euler integration rule is used for the discretization of 5. This gives: obtaining

$$
\begin{aligned}
x[k+1] & =x[k]+\left.h \frac{d x(t)}{d t}\right|_{x=x[k]} \\
& =(1+h A[k]) x[k]+h B u[k]+h d[k] .
\end{aligned}
$$

\section{B. Power Converter Model}

In the experimental setup the motor is driven by a conventional two level voltage source inverter. A scheme of this topology, and the connection to the motor is presented figure 2 .

The different output voltage values generated by this converter are determined by the states of the switches in each of its phases, i.e., by $S_{a}, S_{b}$ and $S_{c}$. Each of these switching functions can adopt values 


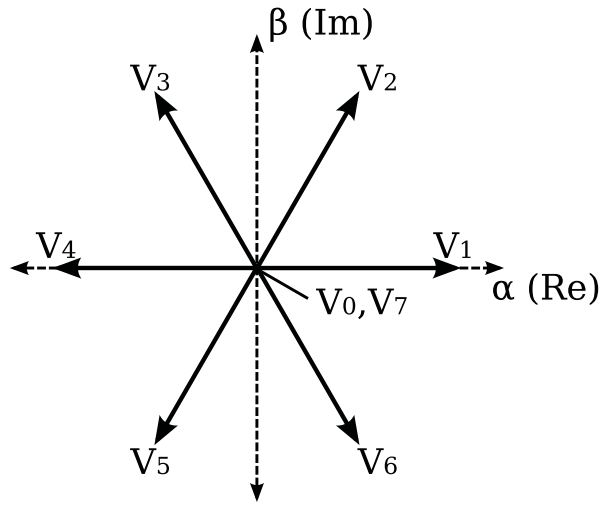

Fig. 3. Voltage vectors generated by the voltage source inverter

$S_{x} \in\{0,1\}$, where $x \in\{a, b, c\}$. The switching state of the inverter can be summarized in a single complex switching function given by

$$
\vec{S} \triangleq \frac{2}{3}\left(S_{a}+\vec{a} S_{b}+\vec{a}^{2} S_{c}\right)
$$

where $\vec{a} \triangleq e^{j 2 \pi / 3}$. This notation allows one to characterize the output voltage vector $\vec{v}_{s}$ as

$$
\vec{v}_{s}=V_{d c} \vec{S}
$$

where $V_{d c}$ is the DC-Link voltage on Fig. 2. Considering all the possible combinations of switching functions $\left(S_{a}, S_{b}, S_{c}\right)$, seven distinct voltage vectors are obtained. These are shown in Fig. 3. These constitute the finite alphabet for the actuation and correspond to the voltage vectors that can be applied to the machine.

The predictive current control strategy is implemented in the rotatory $d q$ frame. Thus, the voltages need to be rotated in order to be used as inputs in 12. In the synchronous $d q$ frame, the voltage vectors become:

$$
\vec{v}_{s}^{(r)}=\vec{v}_{s} e^{-j \theta_{r}} .
$$

\section{Predictive model}

Using the seven possible inverter voltage vectors as inputs to the discrete machine model (12), seven different predictions for the system state in the next sampling instant are obtained.

$$
\begin{aligned}
x_{i}^{p}[k+1]=(1 & +h A[k]) x[k]+h B u_{i}[k]+ \\
& +h d[k], \quad i=0,1, \ldots, 7
\end{aligned}
$$

The superscript $(p)$ denotes predicted values, the subindex $i$ denotes each of the available voltage vectors in the VSI.

These predictions can then be used to evaluate the impact of every voltage vector in the motor electrical variables and the affinity of the predictions with the control goals.

\section{Predictive CurRent Control Scheme For THE PMSM}

As mentioned earlier, the classical control schemes for the PMSM use a cascaded structure, with an external speed controller and an internal current or field control. The proposed scheme preserves this structure, replacing the inner control (PI for FOC, and hysteresis for DTC) with a predictive current controller. This idea is depicted in the drives diagram shown in Fig. 4.

The predictive control algorithm can be understood in three main stages.

- The first stage consists in the prediction of the motor state for every voltage vector available in the inverter. The predicted state is calculated using the discrete model of the drive.

- The second stage consists in the evaluation of a cost function for every predicted state. This cost function is a representation of the control goals.

- Then, the switching state of the inverter is adjusted to apply the selected voltage vector to the machine during the next sampling interval.

\section{A. Cost Function}

A non-negative function that evaluates the distance of the predicted system state to the desired values given by the reference and additional control goals, has to be devised. Such a function is referred to as a cost function, and assumes a minimum value for the predicted state that best agree with the control goals.

In this case, the control goals are:

- Minimization of the torque by ampere ratio, i.e., minimization of the $i_{d}$ current component.

- Tracking of $i_{q}$ current reference.

- Limitation of the stator current amplitude, i.e. maintaining the currents limited.

The cost function designed to achieve this objectives is given by:

$$
\begin{gathered}
F_{c}=\lambda_{i d}\left(i_{s d}^{p}[k+1]\right)^{2}+\lambda_{i q}\left(i_{q}^{p}[k+1]-i_{q}^{*}\right)^{2} \\
+\hat{f}\left(i_{s d}^{p}[k+1], i_{s q}^{p}[k+1]\right)
\end{gathered}
$$

where $i_{q}^{*}$ is the quadrature current reference. The first term of the cost function weights the magnitude of the $i_{d}$ current component. This term penalizes the application of voltage vectors that result in currents that do not generate torque. The second term weights the error between the $i_{q}$ current component and its reference, which is given by the external speed controller. This term penalizes the application of voltage vectors that take the quadrature current away from its reference. The scalars $\lambda_{i d}$ and $\lambda_{i q}$ are weighting factors which are used for tunning purposes. Finally, $\hat{f}$ is a nonlinear 


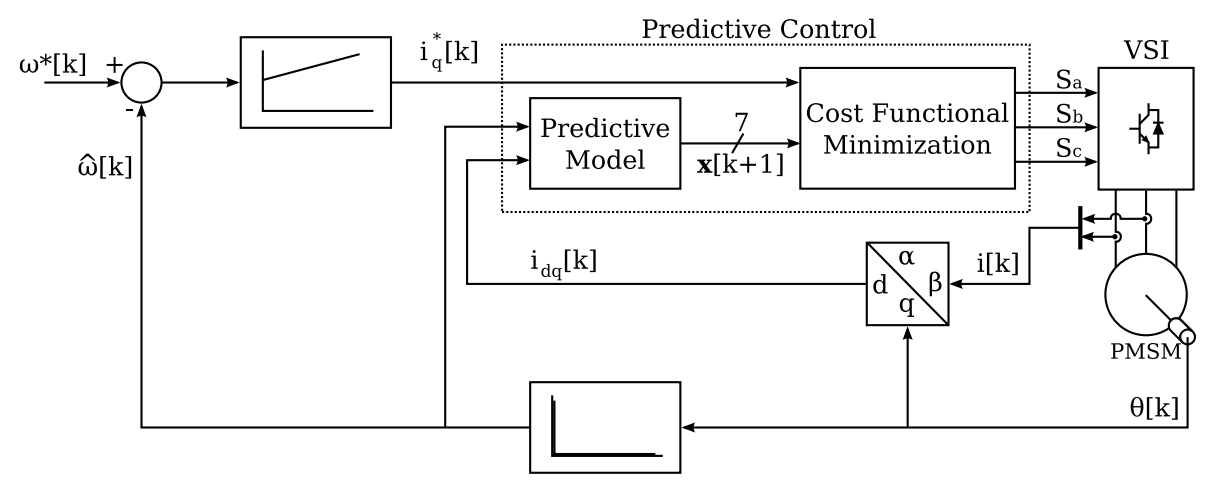

Fig. 4. Proposed Control Scheme for the PMSM.

function given by:

$$
\hat{f}\left(i_{s d}^{p}, i_{s q}^{p}\right) \triangleq \begin{cases}\infty & \text { if }\left|i_{s q}^{p}\right|>\hat{i}_{q} \text { or }\left|i_{s d}^{p}\right|>\hat{i}_{d} \\ 0 & \text { if }\left|i_{s q}^{p}\right| \leq \hat{i}_{q} \text { and }\left|i_{s d}^{p}\right| \leq \hat{i}_{d}\end{cases}
$$

The nonlinear function in 18 strongly penalizes the application of voltage vectors that generate currents which lie outside of an arbitrarily chosen limit. It is included in order to limit the magnitude of both current components.

\section{Simulation Results}

The performance of the proposed control scheme has been tested through simulations using Matlab/Simulink models. The control algorithm was implemented in $\mathrm{C}$ to favor portability. The parameters used in the simulations correspond to those measured in a $4[k W]$ experimental laboratory setup. The results of this simulations are presented in figure 5 , in this figure a startup operation, followed by a speed inversion and a load impact are presented. As can be seen in this figure, current reference tracking is achieved, the $i_{s d}$ current component is minimized, and the current amplitude is effectively limited during acceleration. This show clearly that the proposed strategy achieves simultaneously the three control objectives for which it was devised. It should be noted that fast and completely decoupled control of both current components is achieved, even during the transients.

\section{EXPERIMENTAL RESUltS}

The performance of the proposed control scheme has been tested in an experimental setup, consisting of a $4[k W]$ PMSM and a commercial $7[k W]$ inverter. The machine parameters have been obtained from the nominal values and through testing, and their values are given in table I. The inverter has been properly modified to receive gate signals from an external control platform. In this case, a DSpace 1104 has been used.

In Fig. 6 a speed reverse from $1000[\mathrm{rpm}]$ to $-1000[\mathrm{rpm}]$ is presented. In this figure a startup operation is presented. The performance is very good even in presence of effects that were not taken into account in the simulations and the predictive model, such as semiconductors commutation dead-time, device forward drop, current measurement noise and quantization noise in the angle measurement.

The response to load impact applied by a loading machine coupled to the same shaft of the PSMS, is shown in Fig. 7. The speed reference is set at $1200[\mathrm{rpm}]$. During the load impact, the speed tracking is disturbed as expected. However, the speed PI responds changing the torque current reference, which is followed by the internal control algorithm. This is done without affecting significantly the direct axis current, and hence, the sinusoidal waveform of the line currents are preserved, even during transients.

The current amplitude limit used during the experimental tests was $15[A]$ and $1[A]$ for $\hat{i}_{q}$ and $\hat{i}_{d}$ respectively (eq. 18). As can be seen in figures 6 and 7 , the current amplitude is effectively limited by the inclusion of function 18 in the cost function.

It is important to note that the control of both currents components is still completely decoupled, as expected from simulation results.

TABLE I

MACHINE PARAMETERS.

\begin{tabular}{c|c} 
Parameter & Value \\
\hline \hline$R_{s}$ & $0.3[\Omega]$ \\
$L_{s}$ & $8.2[\mathrm{mH}]$ \\
$\psi_{m}$ & $0.125[\mathrm{~Wb}]$ \\
$p$ & 3 \\
$J$ & $0.004\left[\mathrm{kgm}^{2}\right]$ \\
$B$ & $1 \cdot 10^{-3}\left[\mathrm{kgm}^{2} / \mathrm{s}\right]$
\end{tabular}

\section{CONCLUSIONS}

This paper has presented a new current control algorithm, which does not make use of any linear controller or PWM modulator. When controlled with the proposed scheme, the drive exhibits a very good 

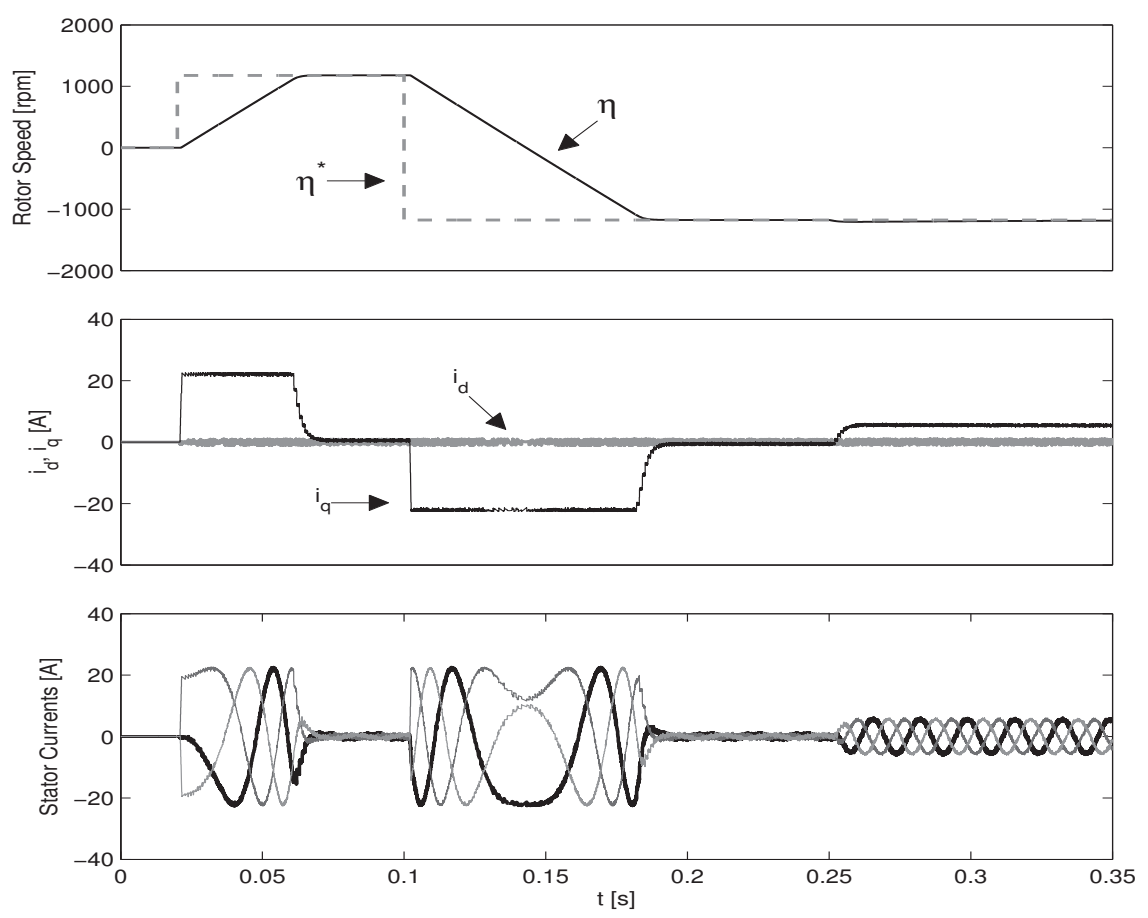

Fig. 5. Simulation results for the operation of the PMSM, using the proposed control scheme.

dynamic and a excellent decoupling between currents $i_{s d}$ and $i_{s q}$ is achieved.

The proposed method is a simple and very attractive alternative to Field Oriented Control and Direct Torque Control for high performance PMSM drives.

\section{REFERENCES}

[1] T. Jahns, G. Kliman, T. Neumann, "Interior Permanent-Magnet Synchronous Motors for Adjustable-Speed Drives," IEEE Trans. Ind. Appl., vol. IA-22, pp. 748-747, 1986.

[2] L. Tang, L. Zhong, M.F. Rahman, Y. Hu, "A novel direct torque control for interior permanent-magnet synchronous machine drive with low ripple in torque and flux - a speed-sensorless approach," IEEE Trans. Ind. Appl., vol. 39, pp. 1748-1756, 2003.

[3] J. Rodríguez, J. Pontt, C. Silva, P. Correa, P. Lezana, P. Cortés, U. Hammann, "Predictive Current Control of a Voltage Source Inverter," IEEE Trans. Ind. Electron., vol. 54, pp. 495-503, 2007.

[4] F. Morel, J. M. Retif, X. Lin-Shi, B. Allard, P. Bevilacqua, "A Predictive Control for a Matrix Converter-Fed Permanent Magnet Synchronous Machine," in Proc. 39th IEEE PESC, Rhodes, Greece, pp. 15-21, 2008.

[5] H. Le-Huy, K. Slimani, P. Viarouge, "Analysis and Implementation of a Real-Time Predictive Current Controller for PermanentMagnet Synchronous Servo Drives," IEEE Trans. Ind. Electron., vol. 41, pp. 110-117, 1994.

[6] M. Pacas, J. Weber, "Predictive Direct Torque Control for the PM Synchronous Machine," IEEE Trans. Ind. Electron., vol. 52, pp. 1350-1356, 2005.

[7] P. Cortés, J. Rodríguez, D. E. Quevedo, C. Silva, "Predictive Current Control Strategy With Imposed Load Current Spectrum," IEEE Trans. Power Electron., vol. 23, pp. 612-618, 2008.

[8] E. Camacho, C. Bordons, Model Predictive Control, Springer, 2004.

[9] W. Leonhard, Control of Electrical Drives, Springer, 1997. 

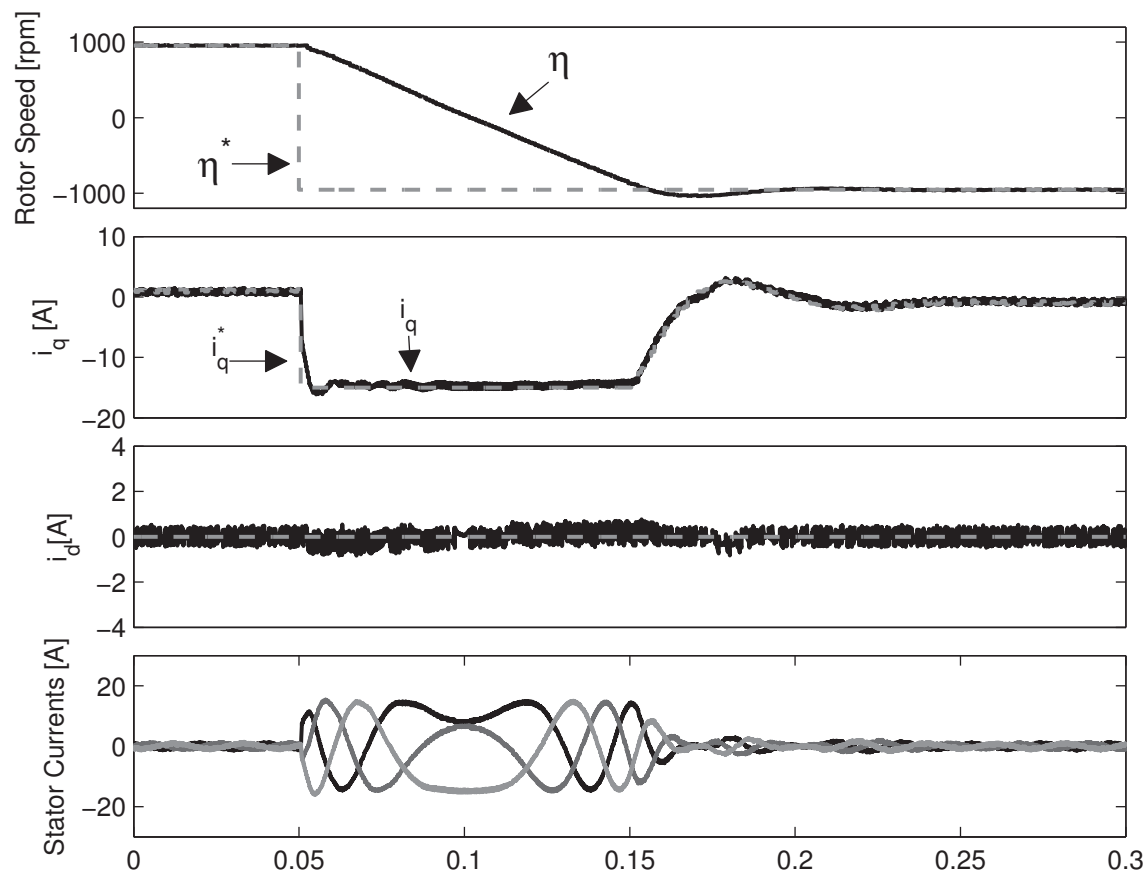

Fig. 6. Experimental results: speed and stator currents during speed reversal operation of the PMSM, using the proposed control method.

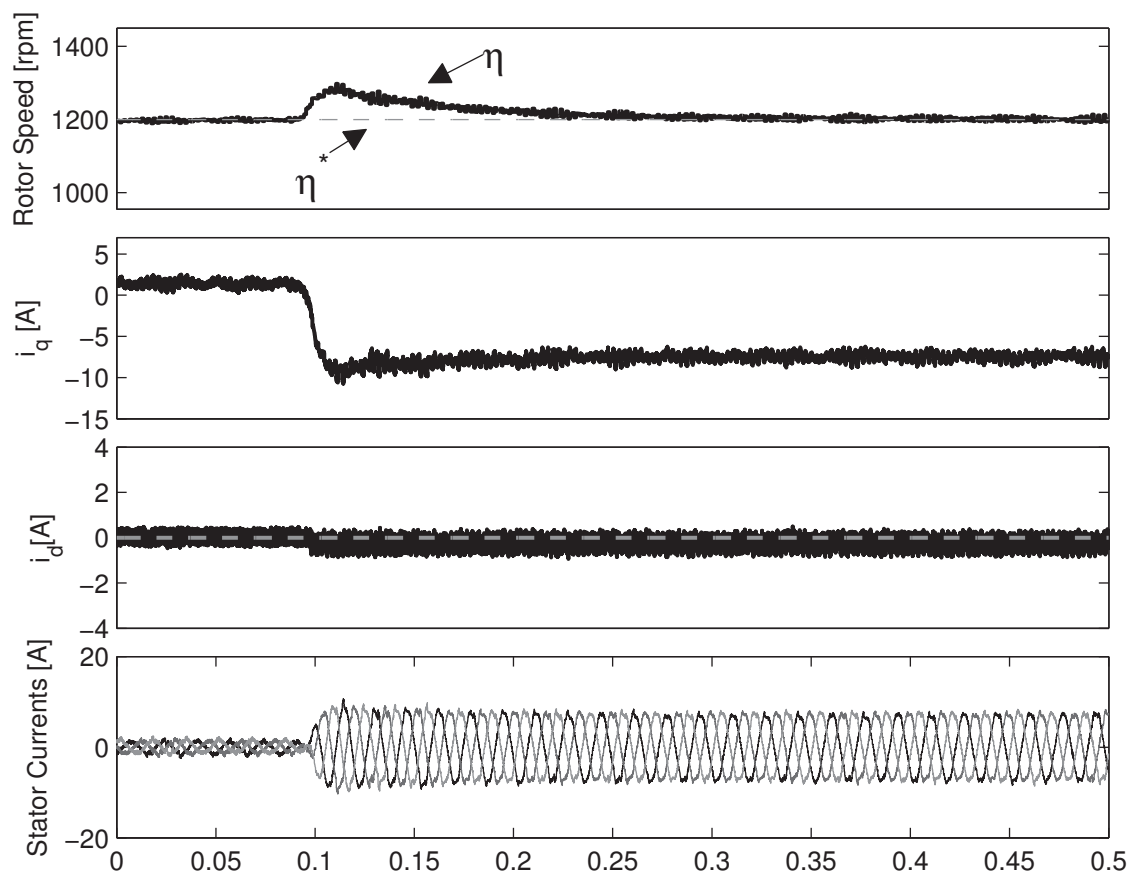

Fig. 7. Experimental results: speed and stator currents during a torque impact operation of the PMSM, using the proposed control method. 